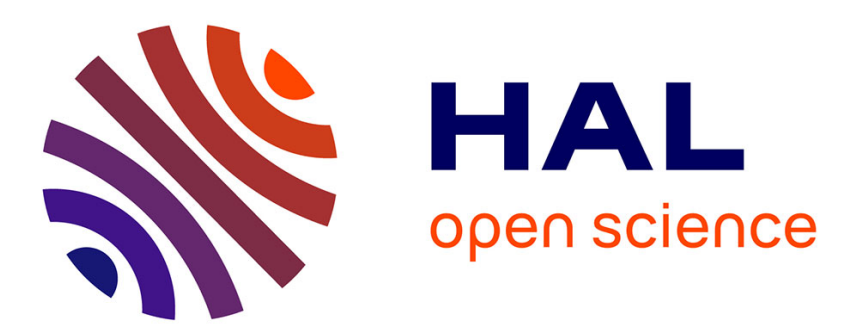

\title{
Hole doping by pressure on the 1111 pnictides CaFeAsF and $\mathrm{SrFeAsF}$
}

Daniele-Cristina Silva-De-Freitas, Gastón Garbarino, Ruben Weht, Amadou

Sow, X. Zhu, F. Han, P. Cheng, J. Ju, H.H. Wen, Manuel Nunez-Regueiro

\section{- To cite this version:}

Daniele-Cristina Silva-De-Freitas, Gastón Garbarino, Ruben Weht, Amadou Sow, X. Zhu, et al.. Hole doping by pressure on the 1111 pnictides CaFeAsF and SrFeAsF. Journal of Physics: Condensed Matter, 2014, 26 (15), pp.155702. 10.1088/0953-8984/26/15/155702 . hal-01002142

\section{HAL Id: hal-01002142 https://hal.science/hal-01002142}

Submitted on 5 Jun 2014

HAL is a multi-disciplinary open access archive for the deposit and dissemination of scientific research documents, whether they are published or not. The documents may come from teaching and research institutions in France or abroad, or from public or private research centers.
L'archive ouverte pluridisciplinaire HAL, est destinée au dépôt et à la diffusion de documents scientifiques de niveau recherche, publiés ou non, émanant des établissements d'enseignement et de recherche français ou étrangers, des laboratoires publics ou privés. 
Hole doping by pressure on the 1111 pnictides $\mathrm{CaFeAsF}$ and $\mathrm{SrFeAsF}$

Daniele C. Freitas ${ }^{1}$, Gastón Garbarino ${ }^{2}$, Ruben Weht ${ }^{3,4}$, Amadou Sow ${ }^{1}$, Xiyu Zhu ${ }^{5}$, Fei Han $^{5}$, Peng Cheng ${ }^{5}$, Jing Ju ${ }^{5}$, Hai Hu Wen ${ }^{5}$, Manuel Núñez-Regueiro ${ }^{1}$

1. Institut Néel, Centre National de la Recherche Scientifique (CNRS) \& Université Joseph Fourier (UJF), 25 Avenue des Martyrs, F-38042 BP166 Grenoble Cedex 9 France

2. European Synchrotron Radiation Facility (ESRF), 6 Rue Jules Horowitz 38043 BP 220 Grenoble

3. Gerencia de Investigación y Aplicaciones, Comisión Nacional de Energía Atómica (CNEA), Avda.

General Paz y Constituyentes, 1650 - San Martín, Argentina

4. Instituto Sabato, Universidad Nacional de San Martín - CNEA, 1650 - San Martín, Argentina

5. National Laboratory for Superconductivity, Institute of Physics and Beijing National Laboratory for

Condensed Matter Physics, Chinese Academy of Sciences, P. O. Box 603, Beijing 100190, China

We determine the pressure phase diagram of the 1111 compounds $\mathrm{CaFeAsF}$ and $\mathrm{SrFeAsF}$, up to $20 \mathrm{GPa}$ and down to $4 \mathrm{~K}$ by electrical resistivity measurements and the change of structure up to $40 \mathrm{GPa}$ at room temperature. The antiferromagnetic transition temperature, as determined by the derivative peak, shows a minimum at $\sim 5 \mathrm{GPa}(10 \mathrm{GPa})$ for the $\mathrm{Ca}(\mathrm{Sr})$ compound. For CaFeAsF, superconductivity appears at this minimum, coincident with the development of a previously reported monoclinic phase. For SrFeAsF, where the orthorhombic and the monoclinic phase were reported to coexist, superconductivity exists above $\mathrm{P} \geq 1 \mathrm{GPa}$. Both phase diagrams can be scaled by a shift of $\sim 10 \mathrm{GPa}$, pressure at which the volume of $\mathrm{SrFeAsF}$ and that of $\mathrm{CaFeAsF}$ at ambient pressure coincide. The difference of our phase diagram with that of electron doped 1111 samples is accounted by hole doping under pressure, that we verified through electron band structure calculations.

Short Title: Hole doping by pressure in 1111 pnictides

PACS: 72.80.Ga, 61.50.Ks, 62.50.-p 
The first discovered iron superconductors[1] were the so-called 1111 phases that are composed of stacked $L n O$ layers and $F e A s$ layers ( $L n$ is a lanthanide) in a tetragonal ( $\mathrm{P} 4 / \mathrm{nmm}) \mathrm{ZrCuSiAs}$ type structure. At temperatures around $150 \mathrm{~K}$, depending on the lanthanide, an orthorhombic ( $\mathrm{Cmma}$ ) distortion develops, followed by an antiferromagnetic state several Kelvin degrees below[2]. The antiferromagnetic state is currently attributed to the development of a spin density wave (SDW), caused by the nesting of the cylindrical hole and electron sheets of the Fermi surface[3]. Electron doping by, e.g. replacement of oxygen by fluorine, leads to the disappearance of both the orthorhombic distortion and the SDW[4], and the emergence of superconductivity. The phase diagram of electron doped 1111 compounds have been largely studied and it seems that there is almost no coexistence between the antiferromagnetic and the superconducting phases, i.e. the transition as a function of doping is apparently of first order [5,6,7]. It has also been shown that hole doping of 1111 compounds can induce superconductivity $[8,9]$. However, there is to date no detailed study of the hole-doped phase diagram of 1111 compounds. Such a study is of central importance, as in cuprates the phase diagram is different for holes and electrons, posing stringent restrictions on possible theories[10]. On the high temperature cuprate superconductors it is accepted that application of pressure is equivalent to hole doping[11]. Simply speaking, the positively charged reservoir layers pump electrons from the negatively $\mathrm{CuO}_{2}{ }^{2-}$ layers as pressure reduces the distance that separates them. In analogy with cuprates, application of pressure has been shown to induce hole doping in the $\mathrm{FeAs}$ planes in the iron superconductor $\mathrm{Sr}_{1-x} \mathrm{~K}_{x} \mathrm{Fe}_{2} A s_{2}$ [12]. Thus one way to study the hole-doped phase diagram would be to apply pressure and follow the different transition temperatures. We report here on coupled transport and structural studies, combined with electronic band structure calculations, of $1111 \mathrm{CaFeAsF}$ and SrFeAsF under pressure.

The CaFeAsF ( $\mathrm{SrFeAsF}$ ) samples were prepared using a two-step solid state reaction method, 
as used for preparing the LaFeAsO samples[13]. In the first step, CaAs ( $\mathrm{SrAs}$ ) was prepared by reacting $C a(\mathrm{Sr})$ flakes (purity $99.9 \%$ ) and $A s$ grains (purity $99.99 \%$ ) at $500{ }^{\circ} \mathrm{C}$ for 8 hours and then $700{ }^{\circ} \mathrm{C}$ for 16 hours. They were sealed in an evacuated quartz tube when reacting. Then the resultant precursors were thoroughly grounded together with $\mathrm{Fe}$ powder (purity 99.95\%) and $\mathrm{FeF}_{3}$ powder (purity $99 \%$ ) in stoichiometry as given by the formula CaFeAsF $(\mathrm{SrFeAsF})$. All the weighing and mixing procedures were performed in a glove box with a protective argon atmosphere. Then the mixture was pressed into pellets and sealed in a quartz tube with an argon atmosphere of 0.2 bar. The materials were heated up to $950{ }^{\circ} \mathrm{C}$ with a rate of $120^{\circ} \mathrm{C} / \mathrm{hr}$ and maintained for 60 hours. Then a cooling procedure to room temperature was followed.

The electrical resistance measurements were performed using a Keithley 220 source and a Keithley 2182 nanovoltmeter. Pressure measurements, $1.4-22 G P a$ (between $4.2 \mathrm{~K}$ and $300 \mathrm{~K}$ ), were done in a sintered diamond Bridgman anvil apparatus using a pyrophillite gasket and two steatite disks as the pressure medium[14]. Pressure cannot be cycled and thus measurements are done only with increasing pressure.

The angle dispersive X-ray diffraction studies on $\mathrm{CaFeAsF}$ and $\mathrm{SrFeAsF}$ powder samples were performed at the ID27 high-pressure beamline of the European Synchrotron Radiation Facility using monochromatic radiation $(\lambda=0.3738 \AA)$ and diamond anvil cells. The transmitting media was nitrogen. The pressure was determined using the shift of the fluorescence line of the ruby. The diffraction patterns were collected with a CCD camera, and the intensity vs. $2 \theta$ patterns were obtained using the fit $2 \mathrm{~d}$ software[15]. A complete Rietveld refinement was done with the GSAS-EXPGUI package[16].

The electronic properties for the different structures were analyzed within the Density Functional Theory (DFT) framework. We used a full-potential linearized augmented plane wave code, Wien2k[17], and GGA[18] to represent the exchange correlation potential. The 
positions for the "heavy" elements were taken directly from the experiments while fluorine coordinates were fixed at their high symmetry positions. We did not do any relaxation or structural minimization.

On Fig. 1 we show the evolution with pressure of the temperature dependence of the electrical resistance of one of the two samples of CaFeAsF. The curves flatten and, above 5GPa show clear signs of appearance of superconductivity, as already reported [19]. The absence of percolation can be attributed to the powder nature of the sample and its sensibility to exposure to humid air during mounting, that causes insulating skins on the sample grains. We define the superconducting onset transition temperature $T_{c}$ by the peak in the resistance. We also show the derivative of the resistance, with a peak that habitually signals the appearance of the antiferromagnetic ordering, $T_{N}$. While at low pressures the peak shifts to lower temperatures with pressure, at higher pressures the tendency is clearly reversed. We have also measured two samples of SrFeAsF with similar results. We plot on Fig. 2 the evolution of both transitions with pressure for all the samples. Differently to previously reported high pressure measurements on 1111 materials ( $\mathrm{LaFeAsO}$ [20], CaFeAsF [19]) we were able to follow the evolution of the transitions for the whole pressure range, without any drop or discontinuity.

A brief description of the pressure temperature structural phase diagram of both compounds has been reported in Ref. [21]. At constant low temperature, both compounds present a transition from orthorhombic to monoclinic (shown on Fig. 2), with a coexistence region between both structures for $S r F e A s F$. On the other hand, for CaFeAsF they find a transition from the high pressure-low temperature monoclinic to the tetragonal phase at $\mathrm{P}=24 \mathrm{GPa}$, $\mathrm{T}=200 \mathrm{~K}$, that falls within error at the extrapolation of our $T_{N}(\mathrm{P})$ curve . If we accept that the peak of the derivative is always associated to $T_{N}$, we can infer that, at high pressures, the antiferromagnetic state coexists with a monoclinic, not orthorhombic phase. Concerning the development of superconductivity under pressure, it approximately coincides with the 
disappearance of the orthorhombic distortion for $C a F e A s F$, similar to what occurs in the electron doped phase diagram. However, it now coexists with a monoclinic distortion, which has not been observed on electron doping. For the $\operatorname{SrFeAsF}$ compound, superconductivity is already detected for the smallest measured pressure, when the sample presumably is in the orthorhombic phase. However, according to Ref. [21], there is a large region of coexistence between the orthorhombic and the monoclinic phase starting at approximately 6GPa. . In high pressure X-ray measurements, a sizeable portion of the sample ( $>10-15 \%)$ must be in the monoclinic phase to be detectable, while superconductivity needs only a small percolation path. It is thus clear that superconductivity will show evidence of a very small portion of the monoclinic phase much earlier in pressure. Thus, the observed phase diagram for $\operatorname{SrFeAsF}$ is not in contradiction with the assumption that there is necessity of a monoclinic phase for the observation of superconductivity. In other words, our measurements seem to indicate that the orthorhombic phase cannot coexist with superconductivity

The structural measurements are shown on the left panel of Fig. 3. At room temperature the evolution of the lattice parameters is smooth and there are no changes as have been reported for other pnictides [22,23]. In particular, the FeAs tetrahedron is always irregular and pressure increases its irregularity. It is interesting to note that the volume of the $\operatorname{SrFeAsF}$ compound coincides with that of the $\mathrm{CaFeAsF}$ at room temperature for a pressure of $10 \mathrm{GPa}$. In fact, both compounds are electronically similar as the alkaline metal do not form bonds present at the Fermi level. It can be assumed that it is the difference in size between the $\mathrm{Sr}$ and $\mathrm{Ca}$ ions that regulates the lattice parameters, the separation between atoms and all the physical properties that depend on them. A way to test this assumption is to shift the pressure axis corresponding to the Sr compound by 10GPa. On the right panel of Fig. 3 we show the effect of such a rigid shift, which verifies the assumption. The differences observed for the superconducting 
regions are probably due to the coexistence of the monoclinic and orthorhombic distortions in SrFeAsF, as discussed above.

Our measurements are not in complete agreement with those of Okada et al. [19]. They measured only $\mathrm{CaFeAsF}$ and their phase diagram shows a brutal disappearance of $T_{N}$ at $4 \mathrm{GPa}$, which we do not observe. It is followed by the sharp appearance of superconductivity at 5GPa, more similar to what we observe. They claim that they follow $T_{N}$ by the derivative of the resistance as we do, but they do not show the corresponding curves. Their work [20] on $\mathrm{LaAsFeO}$ shows a similar brutal disappearance of $T_{N}$ at $12 \mathrm{GPa}$, but with superconductivity in all the pressure range, as we observe in $\operatorname{SrFeAsF}$. The differences may be due to samples, measurement conditions or analysis. Obviously more work on these 1111 materials is necessary to clarify the actual phase diagram.

The phase diagrams that we have obtained are different from those reported for electron doping of 1111 compounds, suggesting that, as is the case for cuprates, pressure is equivalent to hole doping. We have performed calculations of the electronic band structure and of the Lindhard susceptibility $X_{\mathrm{q}}$ at low and high pressures from the atomic positions determined from our structural measurements, Fig. 4. Considering $X_{\mathrm{q}}$, although its magnitude decreases with pressure we do not find any change of position of the peak (as proposed in Ref. [24] for $\mathrm{Fe}_{1+\mathrm{x}} \mathrm{Te}$ ) that, within the SDW scenario, should determine the magnetic ordering vector. However, it does decrease with pressure indicating that the effects of nesting also decrease. Thus, the reason why a monoclinic phase (reported in Ref. 21 ) appears is not due to a change of nesting in our case and the question of the origin of the monoclinic phase remains open. Unfortunately, a determination of the magnetic structure, by e.g. neutron diffraction, would be very difficult with the state-of-the-art experimental means. In any case, it is clear that as, above the minimum of $T_{N}$, as $T_{N}$ increases, $T_{c}$ decreases, implying that both are based on the same parts of the Fermi surface. 
Evidence for hole-doping can be obtained from the evolution of the band structure and charge density at different pressures. We see in Fig. 4, that there is a band along $\Gamma Z$ that gradually dips below the Fermi level as pressure is increased. It bonds the FeAs planes with the CaF planes. As this band absorbs electrons, the Fe-As bands responsible for antiferromagnetism and superconductivity are depleted, and the number of holes in them increases. This appears clearly from the increase in size of the hole cylinders at $\Gamma$. A similar type of phenomena, i.e. non-Fe bands depleting Fe bands under pressure, has been reported [23] in $\mathrm{SmFeAs}_{0.81} F_{0.19}$. Another way to verify this effect is by doing the analysis of the Bader charges [25] corresponding to those planes. In Fig. 5 we plot the corresponding total charge of the Fe-As plane as a function of the applied pressure. As we can see the Fe-As planes become less negative, i.e. doped with holes, with the application of pressure. The electrons flow to the CaF planes, that become more negative.

We can now compare the existing 1111 electron-doped phase diagrams to the hole-doped phase diagrams obtained by pressure. The main difference is that there is apparently coexistence of superconductivity with the antiferromagnetic state, but not with the orthorhombic distortion. It can be argued that the way in which we determine $T_{N}$ is not the optimal one, but in the present state of matters it is the only available. As neutron diffraction is out of question, magnetic susceptibility measurements under pressure are necessary to confirm our results. The fact that a monoclinic to tetragonal transition has been observed at high pressures $\left(\mathrm{P}=24 \mathrm{GPa}, T_{\text {mono } \rightarrow \text { terra }}=200 \mathrm{~K}\right)$ and that it apparently coincides with $T_{N}$ as extrapolated from our results, implies that there is a change of symmetry due to inversion of the order of appearance of the transition temperatures, as predicted theoretically[26]. However, the relation of orthorhombicity and/or monoclinicity with superconductivity remains to be studied. Complete chemical hole doping phase diagrams are needed to clear up these doubts. 
D.C.F. gratefully acknowledges support from the Brazilian agencies CAPES and Cnpq. This work was partially supported by the project TetraFer ANR-09-BLAN-0211 of the Agence Nationale de la Recherche of France. R.W. is fellow of CONICET-Argentina and gratefully acknowledges partial support from CONICET (Grant No. PIP 114-201101-00376) and ANPCyT (Grant No. PICT-2012-0609). 

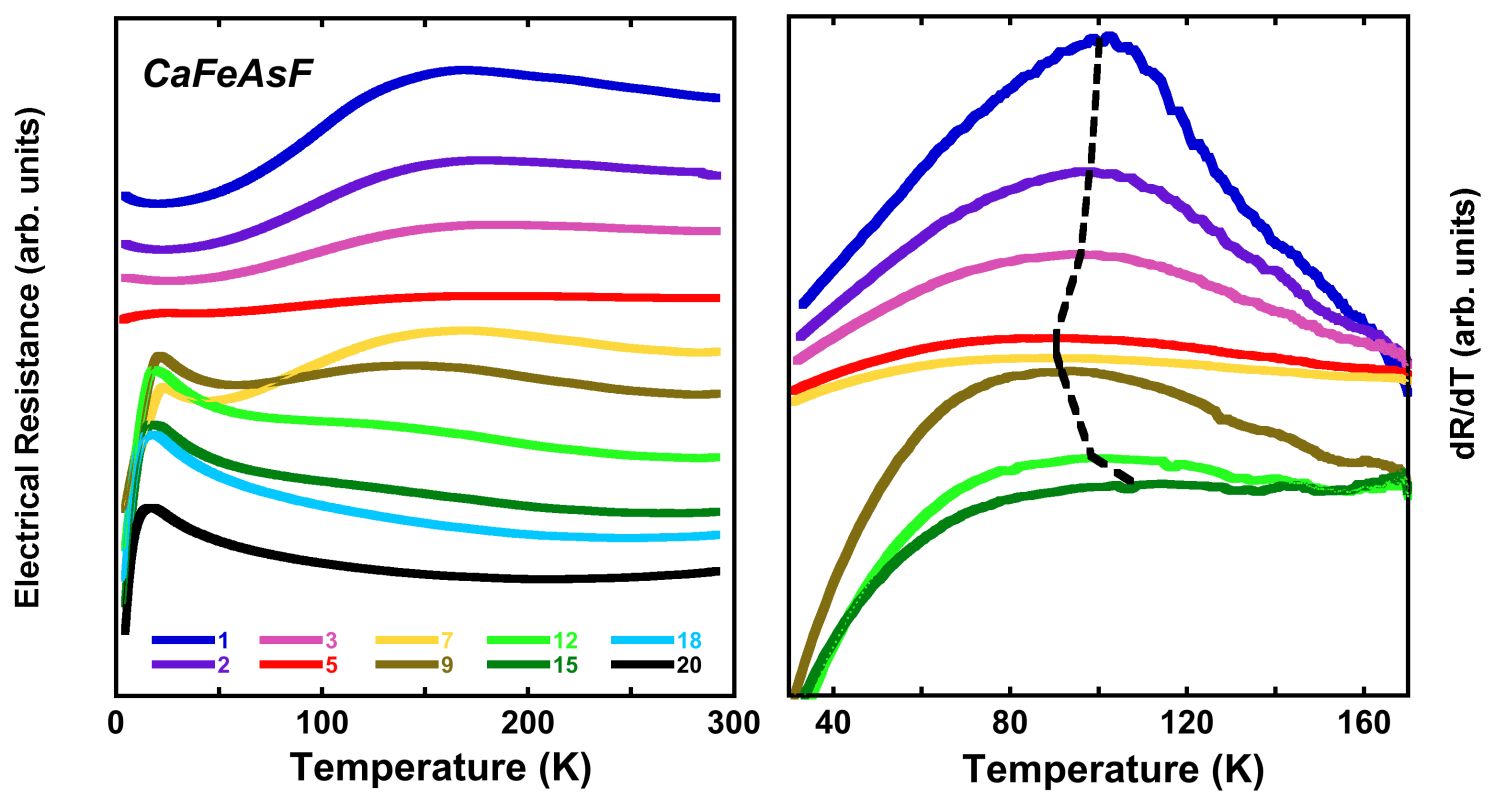

Figure1: (color online) Left Panel: Example of the evolution with pressure of the temperature dependence of the electrical resistance of one sample of $\mathrm{CaFeAsF}$. We observe the gradual appearance of superconductivity above 5 GPa. Right panel: The temperature derivative of the electrical resistance showing how we determine $\boldsymbol{T}_{N}$, the transition temperature to the antiferromagnetic state. The dashed line marks its evolution with pressure; above 18GPa there is no maximum in the curves for this sample. All other samples reported here showed a similar behavior. 

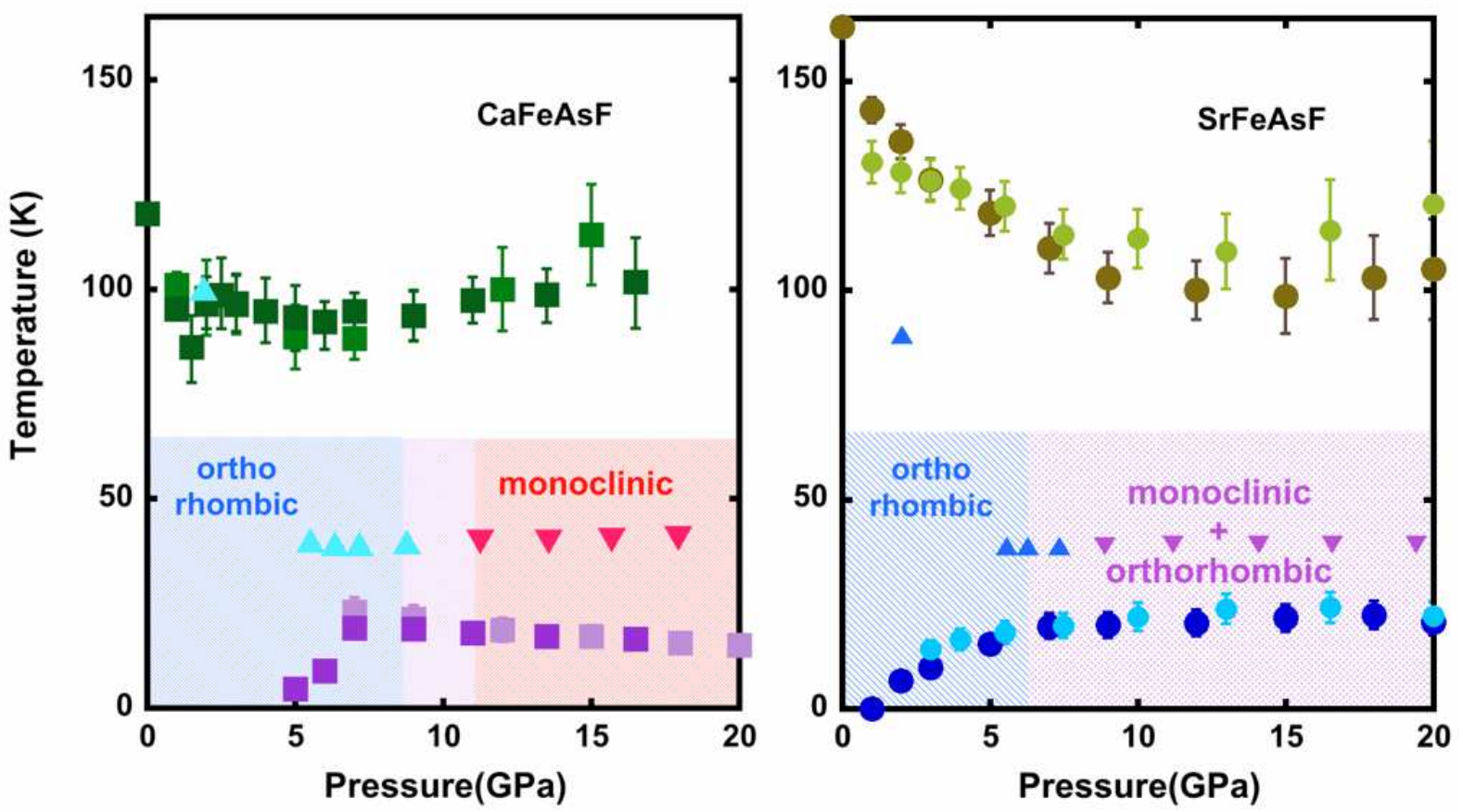

Figure2: (color online)

Left panel: Pressure phase diagram for $\mathrm{CaFeAsF}$, different colors squares correspond to two different samples ( $T_{N}$ green squares; $T_{c}$ magenta squares). The triangles are taken from Ref. 21 and correspond to structural measurements that determine the nature of the distortion at the pressure and temperature of each triangle: orthorhombic (triangles) or monoclinic (inverted triangles). Superconductivity apparently only appears when the orthorhombic distortion disappears.

Right panel: Pressure phase diagram for SrFeAsF, different color circles correspond to two different samples $\left(T_{N}\right.$ green/brown circles; $T_{c}$ blue circles). The triangles are taken from Ref. 21 and indicate orthorhombic (triangles) or mixed orthorhombic-monoclinic (inverted triangles). Superconductivity appears at the lowest measured pressure, there is probably coexistence of the orthorhombic and the monoclinic phase at all pressures, enabling the appearance of superconductivity in the regions where the monoclinic phase has developed (see text). 

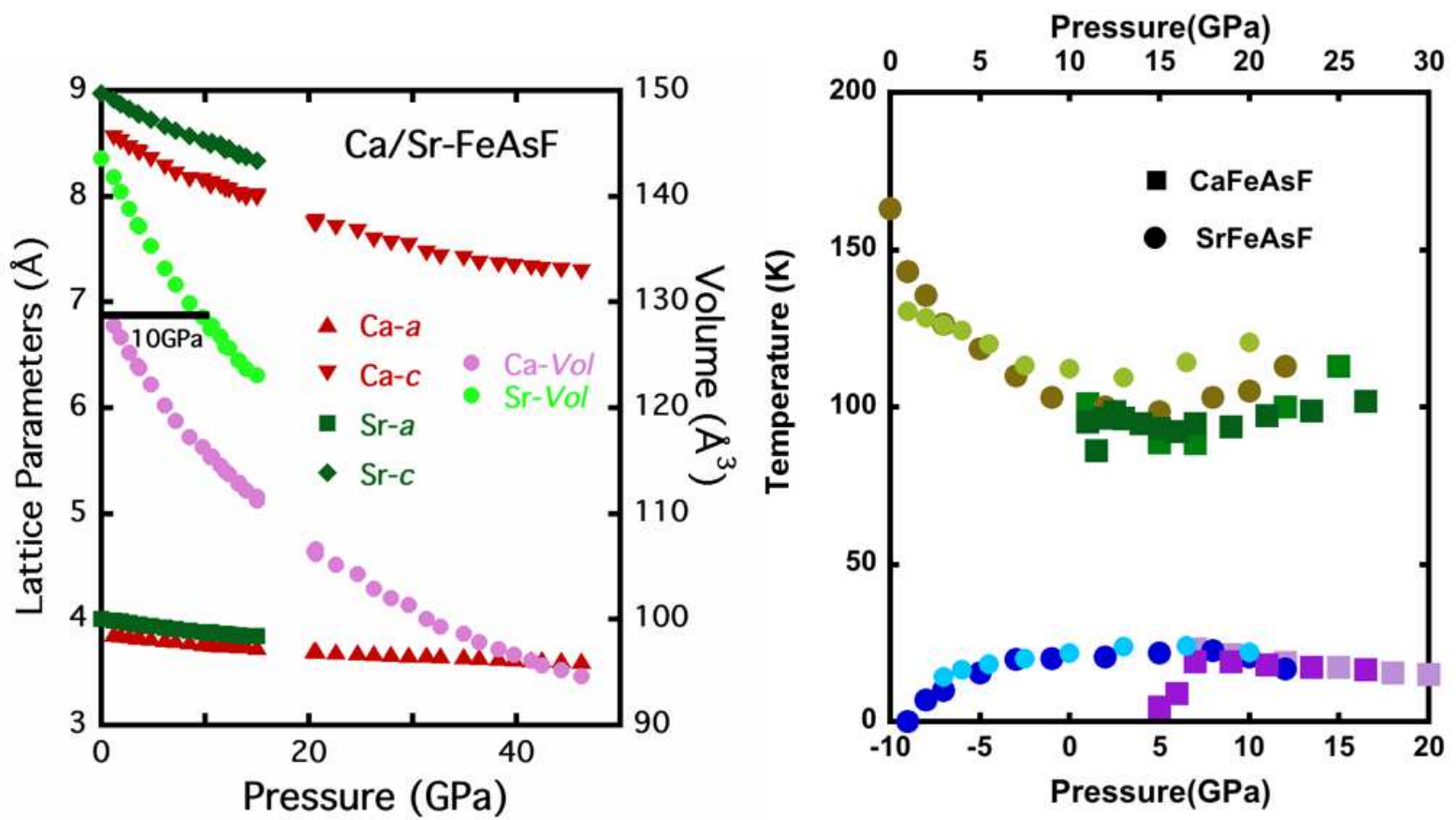

Figure 3: (Color online)

Left panel: Evolution of the lattice parameters of $\mathrm{Ca}$ and $\mathrm{Sr}-\mathrm{FeAsF}$ compounds with pressure. We see that 10GPa separates both dependences, i.e. the volume of the Ca compound becomes the same as that of the Sr compound at 10GPa.

Right panel: Scaling of the phase diagram for the Ca compound on the one of the $\mathrm{Sr}$ compound by a rigid shift of 10GPa (upper scale, lower scale for the $\mathrm{Sr}$ compound). It is clear that the evolution then coincides. However, superconductivity appears suddenly for the Ca compound, which is probably due to no coexistence between the monoclinic and the orthorhombic distortions, due to a sharper transition to the presumed monoclinic phase in this compound (see text). 

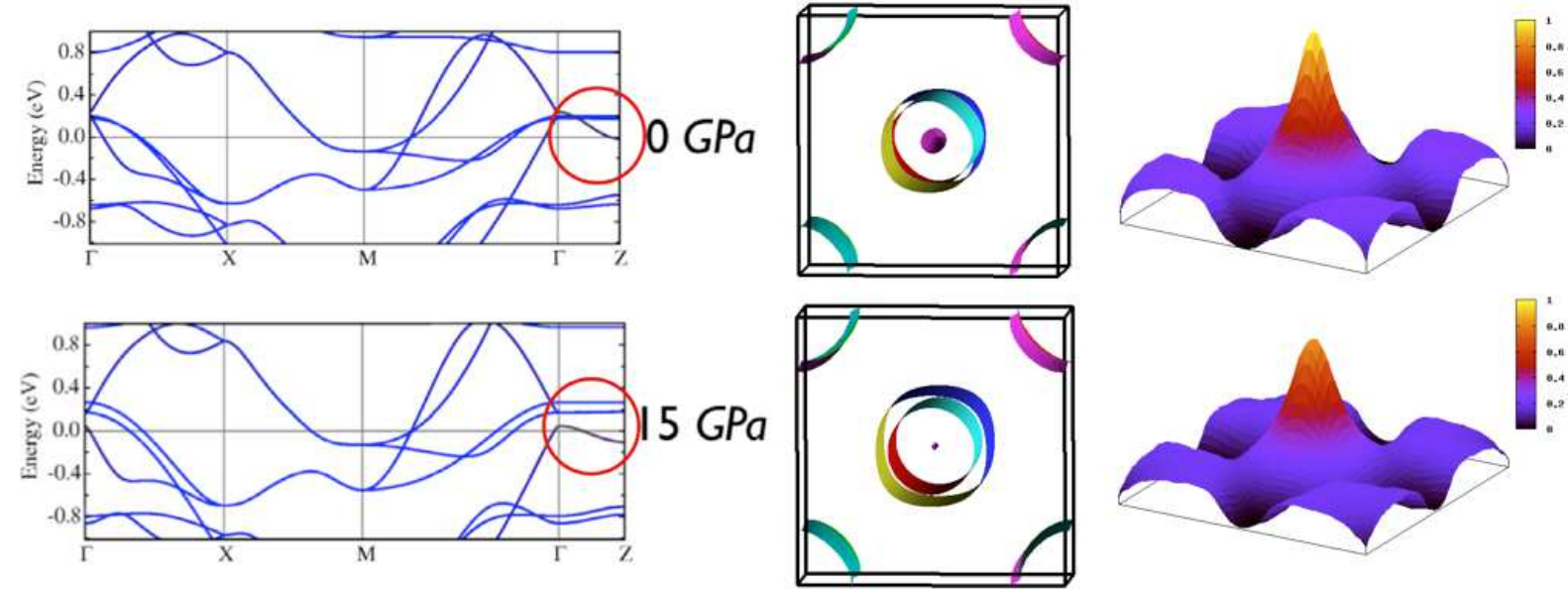

Figure 4: (Color online) Effect of pressure on the electronic band structures. Left panel:

Band structure of $\mathrm{CaFeAsF}$ at two different pressures. The band in magenta between $\Gamma$ and $\mathrm{Z}$ has a smaller $\mathrm{Fe}$ character (inside red circle). Central panel: As pressure increases, the hole barrel at $\Gamma$, which is due to this band, is emptied at the expense of the outer hole cylinder of full $\mathrm{Fe}$ character, i.e. there is an increase of hole doping of the Fe bands under pressure. Right panel: real part of the susceptibility showing that the peak remains at the same $Q$, although its magnitude decreases with pressure. 


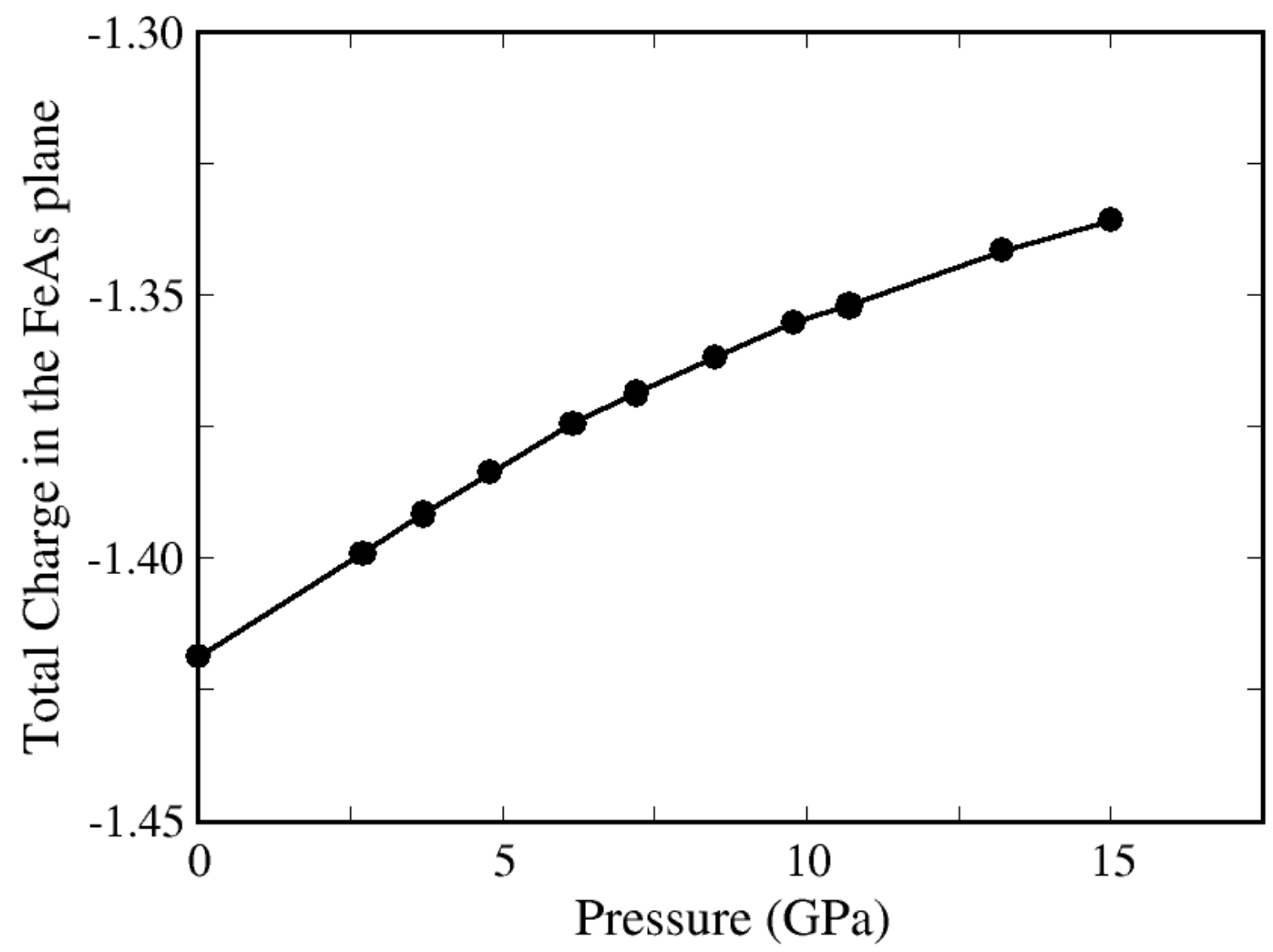

Figure 5: Total charges corresponding to the FeAs planes in function of the applied pressure: As pressure increases charges at these layers become less negative, so there is an effective hole doping of them. Total charges are calculated as the summation of the nuclear and the electronic Bader charges [25] corresponding to the Fe and As atoms. For this task we have used the codes aim (that belongs to the Wien2k package) and critic [27].

[1]Kamihara Y, Watanabe T, Hirano M and Hosono H 2008 J. Amer. Chem. Soc. 1303296 [2]de la Cruz C, et al. 2008 Nature 453899

[3]Maand F, and Lu Z-Y 2008 Phys. Rev. B 78033111

[4] Huang Q, et al. 2008 Phys. Rev. B 78054529

[5]Luetkens H, et al. 2009 Nat. Mat. 8305

[6]Jun Zhao et al. 2008 Nat. Mat. 7953

[7] Rotundu CR, et al. 2009 Phys. Rev. B 80144517

[8] Wen H-H, Mu G, Fang L, Yang H and Zhu X 2008 EPL82 17009

[9]Wen H.H. et al. 2009 Physica C 469894

10 Dagotto E 1994 Rev. Mod. Phys.66 763 
[11] Núñez-Regueiro M and Acha C, in Studies of High Temperaure Superconductors, edited by N.V. Narlikar (Nova Science, New York, 1997), Vol. 24, p.203.

[12] Gooch M Lv, Lorenz B, Guloy A M and Chu C-W 2008 Phys. Rev. B 78180508

[13] Zhu X, Yang H, Fang L, Mu G and Wen H-H 2008 Supercond. Sci. Technol. 21105001

[14] Sanfilippo S et al. 1998 Phys. Rev. B, 61 R3800

[15]Hammersley AP, Svensson SO, Hanfland M, Fitch A.N. and Hausermann D. 1996 High

Pressure Res., 14235

[16]Larson A C and Von Freele 1994 Los Alamos National Laboratory Report LUAR p 86748 (1994); Toby B H 2001 J.Appl.Cryst. 34210

[17]Blaha P, Schwarz K, Madsen G.K.H., Kvasnicka D and Luitz J 2001 in WIEN2K, An Augmented Plane Wave and Local Orbitals Program for Calculating Crystal Properties, edited by Schwarz K (Vienna, University of Technology, Austria,

[18]Perdew J P, Burke K and Ernzerhof M 1996 Phys. Rev. Lett. 773865

[19]Okada H et al. 2011 Phys. Rev.B81 054507

[20]Okada H, Igawa K, Takahashi H, Kamihara Y, Hirano M, Hosono H, Matsubayashi K and Uwatoko Y 2008 J. Phys. Soc. Jpn 77113712

[21]Mishra S K et al. 2011 Phys. Rev. B84 224513

[22] Garbarino G et al., 2011 EPL. 96, 57002

[23] Garbarino G et al. 2011 Phys. Rev. B84 024501

[24] Myung Joon Han and Savrasov S. Y. 2009 Phys. Rev. Lett. 103067001

[25]R. F. W. Bader, 1990, Atoms in Molecules - A Quantum Theory, Oxford University Press, Oxford.

[26] Cano A, Civelli M, Eremin I and Paul I 2010 Phys. Rev. B82 020408

[27]A. Otero-de-la-Roza, M.A. Blanco, A. Martín Pendás and Víctor Luaña, 2009,

Comp.Phys.Commun. 180, 157. 